\title{
Superposition Coding for Power- and Bandwidth Efficient Communication over the Gaussian Channel
}

\author{
Harm S. Cronie ${ }^{* \dagger}$ \\ * Signals and Systems Group \\ University of Twente, $7500 \mathrm{AE}$ Enschede, The Netherlands \\ †Wireless and Mobile Communications Group \\ Delft University of Technology, 2628 CD Delft, The Netherlands \\ Email: h.s.cronie@ewi.utwente.nl
}

\begin{abstract}
A method for power- and bandwidth efficient communication over the Gaussian channel is presented. The method is based on the superposition of binary random variables. As an example we design a signal constellation for a spectral efficiency of $10 \mathrm{bit} / \mathrm{s} / \mathrm{Hz}$. The constellation has a constrained capacity limit only $0.18 \mathrm{~dB}$ from the Gaussian capacity limit. Moreover, we show that combined with multi-level coding and low-density parity-check codes, a low bit-error rate is achieved at $0.6 \mathrm{~dB}$ from the Gaussian capacity limit for a large codeword length.
\end{abstract}

\section{INTRODUCTION}

In this paper we consider power- and bandwidth efficient communication over the additive white Gaussian noise (AWGN) channel. The goal is to achieve reliable communication at a rate close to the capacity of the channel for high spectral efficiencies where the use of binary signaling incurs a large loss in rate. In this case one has to resort to signal shaping methods to get close to capacity. A restriction to signal constellations with a uniform spacing and an equiprobable selection of the constellation symbols leads to a maximum loss of $1.53 \mathrm{~dB}$ compared to a Gaussian channel input [1].

Power- and bandwidth efficient communication with signal shaping has been studied by several authors. Most methods are based on multi-dimensional signal constellations [2], [3] or non-uniform signaling and non-equiprobable signaling methods [4], [5]. For a more extensive overview of coding and modulation for Gaussian channels we refer to [1]. Some recent research has focussed on the combination of powerful binary error-correcting codes and signaling methods. See e.g. [6], [7] and references herein.

In this paper we present a simple modulation method which allows to generate signal constellations with a nonuniform spacing and a non-equiprobable distribution on the constellation symbols. Furthermore, the method is easily combined with binary error-correcting codes to provide reliable communication. The outline of the paper is as follows. In Section II we introduce the modulation method and show how to combine it with binary block codes. In Section III we consider the design of signal constellations and present an example of a large signal constellation which allows us to transmit at $10 \mathrm{bit} / \mathrm{s} / \mathrm{Hz}$ close to the capacity limit of the AWGN channel. Furthermore, we design binary low-density paritycheck (LDPC) codes optimized for this signal constellation and present simulation results in Section IV. We end with conclusions in Section V.

\section{Modulation AND CODING}

We consider power- and bandwidth efficient communication over the AWGN channel. This channel is defined by

$$
Y=X+N
$$

where the channel input $X$ is disturbed by the random variable $N$ which has a zero-mean Gaussian distribution with variance $\sigma^{2}$. The capacity of the channel is achieved for a Gaussian input and is given by the well-known formula

$$
C=\frac{1}{2} \log _{2}\left(1+\frac{E_{s}}{\sigma^{2}}\right) \quad \text { bit/use, }
$$

where $E_{s}$ is the average symbol energy.

\section{A. Modulation}

To get close to capacity for high signal-to-noise ratios, the input and output distribution of the AWGN channel should be near-Gaussian. One way to generate a Gaussian distribution is by adding independent and identically-distributed (i.i.d.) random variables. Suppose we generate a constellation symbol $Z$ by adding $d$ i.i.d. uniform random bit variables

$$
Z=\frac{1}{\sqrt{d}} \sum_{i=1}^{d} X_{i}
$$

where $X_{i}$ is a binary uniform distributed random variable taking values -1 and 1 . The resulting distribution of $Z$ is binomial and when we let $d \rightarrow \infty$ the distribution of $Z$ converges to the Gaussian distribution by the central limit theorem. Now, let $X_{1}, \ldots, X_{d}$ represent a tuple of bits from a memoryless bit source where each bit takes values -1 and 1 with equal probability. Furthermore, we can scale each of the $X_{i}$ by a positive constant $\alpha_{i}$ before addition. The achievable shaping gain depends on the values of the $\alpha_{i}$. A constellation symbol is generated by

$$
Z=\sum_{i=1}^{d} \alpha_{i} X_{i}
$$

Depending on the $\alpha_{i}$ the map from $X_{1}, \ldots, X_{d}$ to a constellation symbol is many-to-one or one-to-one. Furthermore, 


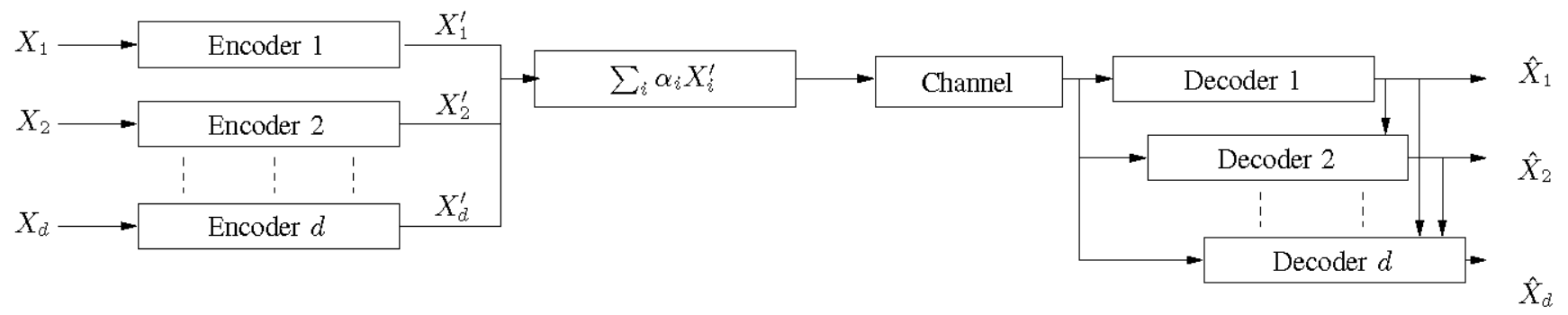

Figure 1: The modulation method with multi-level coding and multi-stage decoding.

equation 4 not only determines the constellation symbols, but also the mapping from bits to constellation symbols.

The idea of superimposing bits is not new. In [8] multilevel coding is introduced where the output of $d$ independent binary encoders is summed. Moreover, in [9] and [10] the authors show that for small values of $d$ and low spectral efficiencies the method can be combined with turbo codes to lead to a performance within $1.4 \mathrm{~dB}$ of the capacity of the AWGN channel. In this paper we follow the same approach, but consider moderate to high spectral efficiencies and combine the modulation method with LDPC codes.

\section{B. Multilevel coding with multi-stage decoding}

To derive a scheme which allows us to use binary errorcorrecting codes we consider the mutual information between $X_{1}, \ldots, X_{d}$ and the channel output $Y$

$$
\begin{array}{r}
I\left(\left(X_{1}, \ldots, X_{d}\right) ; Y\right)=I\left(X_{1} ; Y\right)+I\left(X_{2} ; Y \mid X_{1}\right)+\ldots+ \\
I\left(X_{d} ; Y \mid X_{1}, \ldots, X_{d-1}\right),
\end{array}
$$

where we have used the chain rule of mutual information. We encode each of the $X_{i}$ by a binary error-correcting code. This is the multi-level coding (MLC) procedure [8], [11]. At the receiver we can use a multi-stage decoding (MSD) procedure where the sequence of $X_{1}$ bits is decoded first and the decision is passed on to the next decoder which decodes the sequence of $X_{2}$ bits. This procedure continues up to the last level. An overview of this system is shown in figure 1. Note that a joint decoding procedure is possible as well [12]. However, in this case the design of error-correcting codes is less straightforward.

\section{Binary subchannels}

In the MLC-MSD scheme each bit is transmitted through an equivalent binary-input real-output channel. At level $l$ the values of $X_{1}, \ldots, X_{l-1}$ are assumed to be known and can be subtracted from the channel output. The channel output at level $l$ becomes

$$
Y_{l}=Y-\sum_{i=1}^{l-1} \alpha_{i} X_{i}=\sum_{i=l}^{d} \alpha_{i} X_{i}+N
$$

At level $l$ bit $X_{l+1}, \ldots, X_{d}$ are considered to be noise. Thus for bit $X_{l}$ the channel takes the form

$$
Y_{l}=\alpha_{l} X_{l}+N_{l}
$$

where

$$
N_{l}=\sum_{i=l+1}^{d} \alpha_{i} X_{i}+N
$$

and has density $f_{N_{l}}$

$$
f_{N_{l}}(n)=\frac{1}{2^{d-l} \sqrt{2 \pi \sigma^{2}}} \sum_{x_{l}+1} \ldots \sum_{x_{d}} e^{\left.-\frac{\left(n-\alpha_{l+1} x_{l}+1\right.}{2 \sigma^{2}}-\ldots-\alpha_{d} x_{d}\right)^{2}}
$$

This density satisfies

$$
f_{N_{l}}(n)=f_{N_{l}}(-n)
$$

which implies that the channel transition probability density function $f_{Y_{l} \mid X_{l}}$ at level $l$ satisfies

$$
f_{Y_{l} \mid X_{l}}\left(y \mid x_{l}\right)=f_{Y_{l} \mid X_{l}}\left(-y \mid-x_{l}\right) .
$$

In other words, the equivalent channel is a binary memoryless symmetric (BMS) channel. In the next sections we consider the design of signal constellations and error-correcting codes.

\section{Design of Signal Constellations}

In this section we consider the design of signal constellations which can be generated by equation 4 . We investigate how the $\alpha_{i}$ can be chosen such that $I\left(\left(X_{1}, \ldots, X_{d}\right) ; Y\right)$ is close to the capacity of the AWGN channel. We consider conventional pulse-amplitude modulation (PAM) signal constellations, binomial signal constellations and numerically optimized signal constellations. For the latter we consider a high spectral efficiency of $5 \mathrm{bit} / \mathrm{use}$ or $10 \mathrm{bit} / \mathrm{s} / \mathrm{Hz}$.

\section{A. PAM signal constellations}

Signal constellations with a uniform spacing and a uniform distribution on the constellation symbols can be generated by choosing the $\alpha_{i}$ as consecutive powers of two. A constellation symbol $Z$ is generated by

$$
Z=\sum_{i=1}^{d} 2^{i-1} X_{i}
$$

In this case the modulation method results in a natural labeling of the constellation symbols. Although interesting, we do not consider these conventional constellations any further in this paper. 


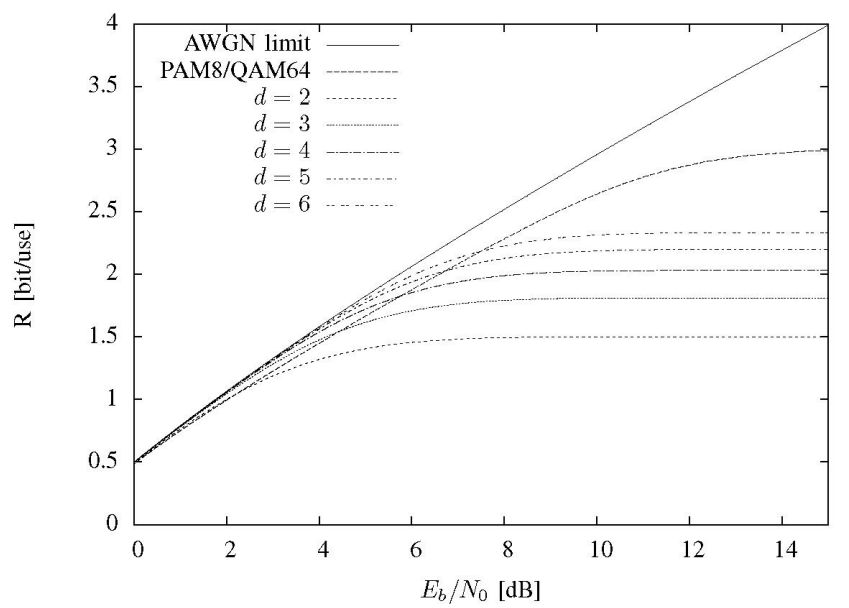

Figure 2: The constrained capacity of the binomial signal constellations.

\section{B. Binomial signal constellations}

An interesting family signal constellations is generated when each of the $\alpha_{i}$ is chosen as 1. A constellation symbol $Z$ is generated by

$$
Z=\sum_{i=1}^{d} X_{i}
$$

This map is many-to-one and a constellation symbol is selected with probability

$$
\mathrm{P}(Z=2 i-d)=\left(\begin{array}{c}
d \\
i
\end{array}\right) 2^{-d}, \quad \text { for } i=0, \ldots, d
$$

This distribution is the binomial distribution. The constrained constellation capacity of these signal constellations is shown in figure 2. We observe that depending on the SNR, the constrained capacity is very close to the unconstrained capacity.

This family of constellations is only useful for low to moderate spectral efficiencies, because at high spectral efficiencies a large $d$ is required. The reason for this is that the entropy of the binomial distribution grows at most logarithmically with $d$.

\section{Numerically optimized signal constellations}

An additional advantage of using equation 4 to generate a signal constellation is that a relatively few degrees of freedom determine the constellation geometry and mapping from bits to constellation symbols. Numerical optimization becomes feasible. To illustrate the concept, we target a spectral efficiency of $5 \mathrm{bit} / \mathrm{use}$ or $10 \mathrm{bit} / \mathrm{s} / \mathrm{Hz}$. For a Gaussian channel input this rate is achieved when $E_{s} / \sigma^{2}=1023$. The constellation has to support the spectral efficiency and this requires that $d \geq 5$. As an example we consider $d=8$ in this paper.

We design a signal constellation for $d=8$ by optimizing the values of the $\alpha_{i}$ by a numerical maximization of the mutual information between the channel input and the channel output given that we generate the channel inputs according to equation 4. Moreover, we incorporate a power constraint by setting $E_{s}=1$ which in terms of the $\alpha_{i}$ corresponds to $\sum_{i=1}^{8} \alpha_{i}^{2}=1$. At $\sigma^{2}=1 / 1023$ the result of the optimization is as follows

$$
\begin{array}{r}
Z=0.5107 X_{1}+0.3986 X_{2}+0.3475 X_{3}+0.2591 X_{4} \\
+0.1498 X_{5}+0.0634 X_{6}+0.5260 X_{7}+0.2989 X_{8}
\end{array}
$$

The resulting signal constellation has 256 symbols and a nonuniform spacing of the constellation symbols. Figure 3 shows the quadrature constellation which is generated by using each dimension independently. Equation 15 determines the constellation geometry and the mapping from bits to constellation symbols. Figure 4 shows the constrained capacity of the signal constellation plotted as a function of $E_{b} / N_{0}$ together with the constrained capacity of a pulse amplitude modulation (PAM) constellation with 256 symbols. We observe that at $\sigma^{2}=$ $1 / 1023$ the constrained capacity of the constellation is 4.97 bit/use. In terms of $E_{b} / N_{0}$ the distance to the capacity of the AWGN channel is only $0.18 \mathrm{~dB}$. The PAM 256 constellation requires an additional $1.24 \mathrm{~dB}$ to achieve the same rate. The signal constellation achieves a shaping gain of $1.24 \mathrm{~dB}$. The figure also shows the capacities of the binary subchannels whose sum is equal to the total capacity.

\section{DESIGN OF ERROR-CORRECTING CODES}

\section{A. Low-Density Parity-Check Codes}

The signal constellation generated by equation 15 leads to a set of binary memoryless symmetric (BMS) channels in the MLC-MSD scheme if we assume perfect cancellation. In [13] it is shown that for several BMS channels LDPC codes can be designed which perform very well and in this section we

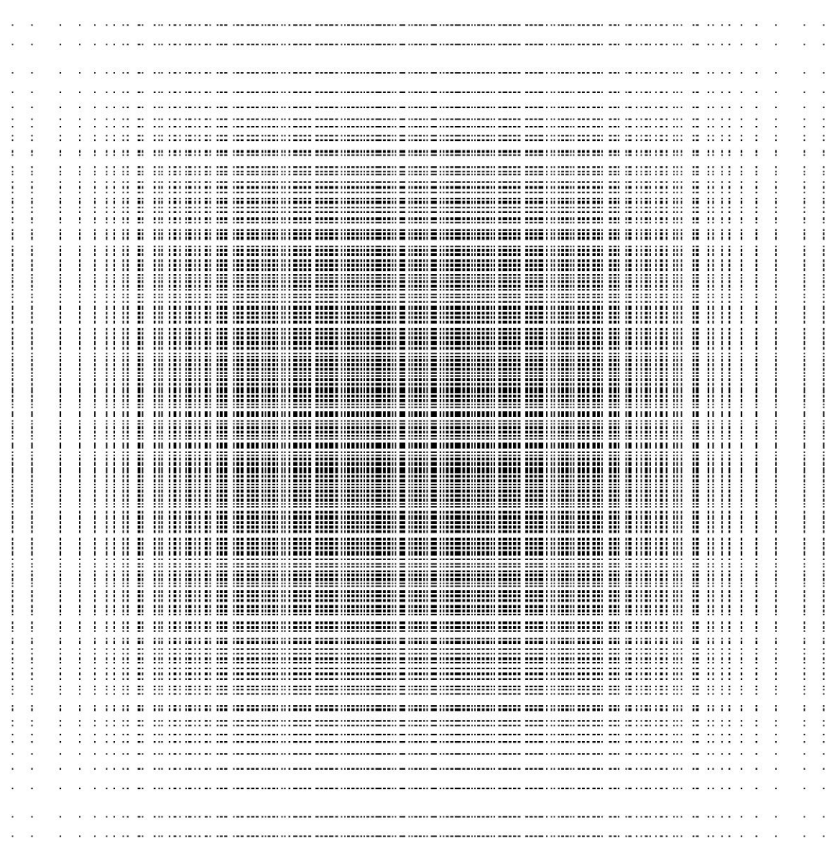

Figure 3: The quadrature signal constellation with 65536 symbols. 


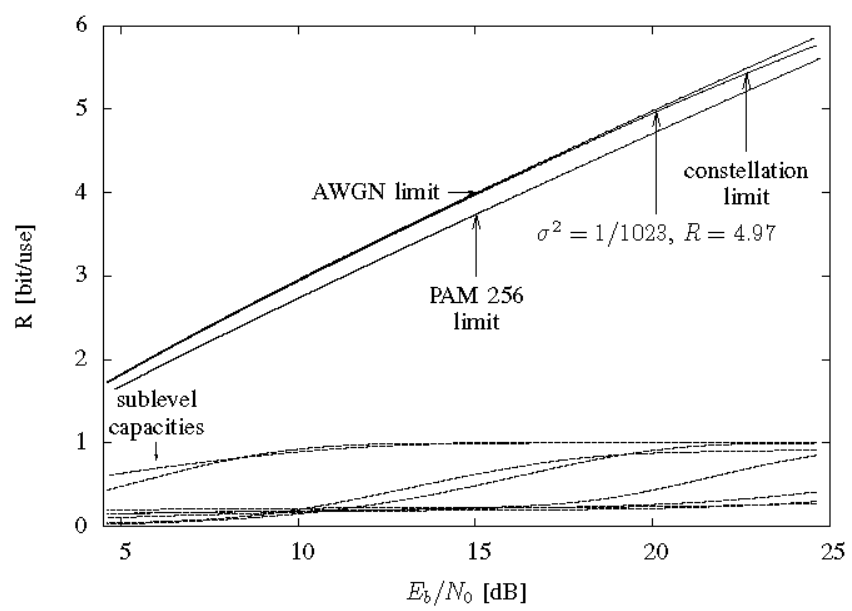

Figure 4: The capacity limit of the numerically optimized signal constellation.

show that this also holds for the BMS channels defined by the signal constellation.

We have designed LDPC codes for the channels of bit $X_{1}, \ldots, X_{6}$ by a method based on density evolution which is similar as described in [13]. The channels for $X_{7}$ and $X_{8}$ are used uncoded, because their uncoded bit error rate is sufficiently low. For multilevel coding schemes the design of the code rates is an issue and one can use rate design techniques as described in [11]. We design LDPC codes for a noise variance of $\sigma^{2}=1 / 1023$ and a target block length of 32000 per level. We use the notation of [13] to specify the LDPC codes and the degree distributions for the variable nodes for level one to six are given by:

$\lambda_{1}(x)=0.362 x+0.300 x^{2}+0.125 x^{7}+0.087 x^{8}+0.126 x^{19}$

$\lambda_{2}(x)=0.362 x+0.279 x^{2}+0.108 x^{6}+0.055 x^{7}+0.196 x^{19}$

$\lambda_{3}(x)=0.336 x+0.270 x^{2}+0.154 x^{6}+0.034 x^{7}+0.206 x^{19}$

$\lambda_{4}(x)=0.277 x+0.234 x^{2}+0.061 x^{5}+0.163 x^{6}+0.265 x^{19}$

$\lambda_{5}(x)=0.196 x+0.240 x^{2}+0.235 x^{6}+0.002 x^{7}+0.327 x^{19}$

$\lambda_{6}(x)=0.147 x+0.261 x^{2}+0.215 x^{6}+0.081 x^{7}+0.221 x^{18}$

$$
+0.075 x^{19}
$$

The checknode distributions are right-concentrated with average degrees of $4,4,4.4,6.3,32$ and 46 for level one to six, respectively. The rates of the codes for level one to six are equal to $0.201,0.184,0.228,0.397,0.863$ and 0.900 , respectively. This gives a total rate of $4.77 \mathrm{bit} / \mathrm{use}$ when we include the two uncoded levels.

Figure 5 shows the simulation results for transmission over the AWGN channel for a block length of 32000 . The LDPC codes are decoded by the sum-product algorithm. The figure shows the performance of the individual codes and the total average performance. In the first case, the simulation is performed with perfect decisions at the higher levels, i.e. there is no error propagation between different levels. In the second case, error propagation is taken into account. We observe that once the bit-error rate (BER) has reached a sufficiently low value, error propagation hardly plays a role. If we compare the performance of the levels, we observe that the behavior of the binary channels of the levels is different. This is a consequence of the difference in the noise structure of these binary channels. An average BER of $10^{-4}$ is achieved at an $E_{s} / \sigma^{2}$ of $30 \mathrm{~dB}$, which corresponds to a distance of $1.14 \mathrm{~dB}$ to the constellation capacity limit.

We also designed a set of degree distributions for a block length of 320000 and the degree distributions for the variable

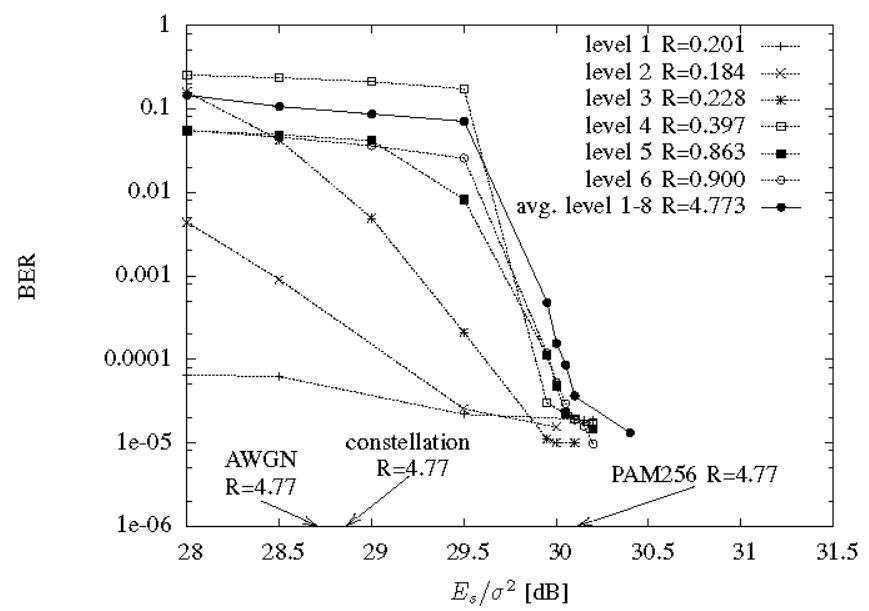

Figure 5: Simulation results for codeword length 32000.

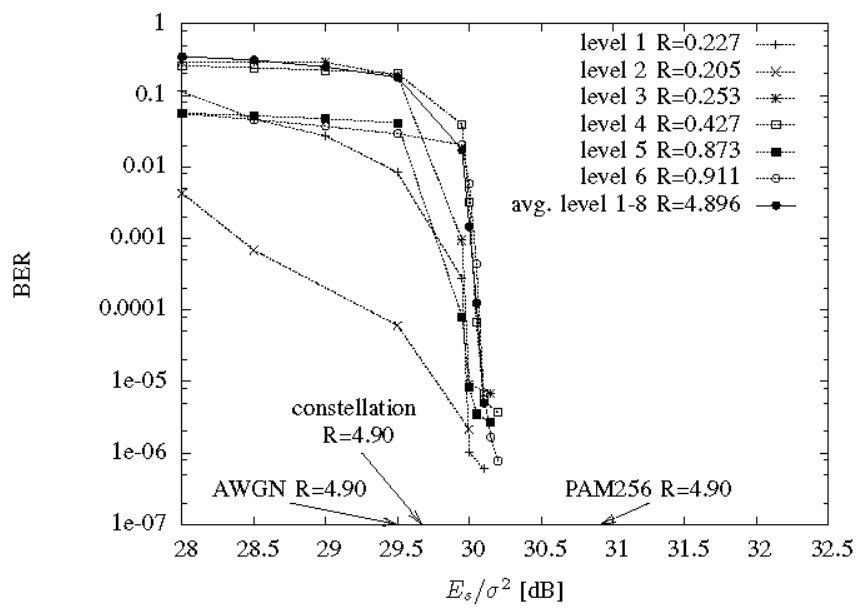

Figure 6: Simulation results for codeword length 320000. 
nodes for level one to six are given by:

$$
\begin{array}{r}
\lambda_{1}(x)=0.288 x+0.255 x^{2}+0.159711 x^{7}+0.071 x^{8} \\
+0.097 x^{23}+0.0153 x^{25}+0.114 x^{49} \\
\lambda_{2}(x)=0.291 x+0.250 x^{2}+0.126 x^{7}+0.103 x^{8} \\
+0.069 x^{19}+0.161 x^{49} \\
\lambda_{3}(x)=0.281 x+0.176 x^{2}+0.166 x^{5}+0.017 x^{10} \\
+0.116 x^{11}+0.243 x^{49} \\
\lambda_{4}(x)=0.218 x+0.200 x^{2}+0.187 x^{6}+0.009 x^{7} \\
+0.059 x^{13}+0.0764 x^{14}+0.249 x^{49} \\
\lambda_{5}(x)=0.166 x+0.181 x^{2}+0.174 x^{6}+0.039 x^{7} \\
+0.130 x^{15}+0.019 x^{17}+0.292 x^{49} \\
\lambda_{6}(x)=0.104 x+0.191 x^{2}+0.019 x^{3}+0.173 x^{8} \\
+0.136 x^{9}+0.377 x^{49}
\end{array}
$$

The code rates of level one to six are slightly higher and given by $0.227,0.205,0.253,0.427,0.873$ and 0.911 , respectively. Furthermore, the maximum variable node degree of the LDPC codes is 50 instead of 20 . In this case the total rate at which we transmit is 4.90 and the simulation results are shown in figure 6. A BER $<10^{-5}$ is achieved at $0.6 \mathrm{~dB}$ from the capacity of the AWGN channel. This BER is achieved far before the PAM256 capacity limit which shows that with a PAM256 constellation one can never achieve the same performance.

\section{CONCLUSIONS}

We have presented a modulation method which allows to design signal constellations which have a constrained capacity close to the capacity of the AWGN channel. As an example we have designed a signal constellation for $10 \mathrm{bit} / \mathrm{s} / \mathrm{Hz}$ which combined with LDPC coding leads near-capacity performance. For a large block length, a low bit-error rate is achieved at only $0.6 \mathrm{~dB}$ from the capacity of the AWGN channel at a transmission rate of $9.8 \mathrm{bit} / \mathrm{s} / \mathrm{Hz}$.

\section{ACKNOWLEDGMENTS}

The author wishes to thank R.L. Urbanke for useful discussions while visiting EPFL, Lausanne and C.H. Slump for reviewing an initial version of this paper and J.H. Weber for reviewing the final version.

\section{REFERENCES}

[1] G. D. Forney, Jr. and G. Ungerboeck, "Modulation and coding for linear Gaussian channels," IEEE Trans. Inform. Theory, vol. 23, pp. 2384 2415, Oct. 1998.

[2] G. D. Forney, Jr. and L.-F. Wei, "Multidimensional constellations-part I: Introduction, figures of merit, and generalized cross constellations," IEEE J. Select. Areas Commun., vol. 7, pp. 877-892, Aug. 1989.

[3] U. Erez and R. Zamir, "Achieving $1 / 2 \log (1+$ SNR) on the AWGN channel with lattice encoding and decoding," IEEE Trans. Inform. Theory, vol. 50, pp. 2293-2314, Oct. 2004.

[4] F. R. Kschischang and S. Pasupathy, "Optimal nonuniform signaling for Gaussian channels," IEEE Trans. Inform. Theory, vol. 39, pp. 913-929, May 1993.

[5] A. R. Calderbank and L. H. Ozarow, "Nonequiprobable signaling on the Gaussian channel," IEEE Trans. Inform. Theory, vol. 36, pp. 726-740, July 1990

[6] J. Hou, P. H. Siegel, L. B. Milstein, and H. D. Pfister, "Capacityapproaching bandwidth-efficient coded modulation schemes based on low-density parity-check codes," IEEE Trans. Inform. Theory, vol. 49, pp. 2141-2155, Sept. 2003.

[7] P. Limpaphayom and K. A. Winick, "Power- and bandwidth-efficient communications using LDPC codes," IEEE Trans. Commun., vol. 52, pp. 350 - 354, Mar. 2004.

[8] H. Imai and S. Hirakawa, "A new multilevel coding method using errorcorrecting codes," IEEE Trans. Inform. Theory, vol. 23, pp. 371-377, May 1977.

[9] L. Duan, B. Rimoldi, and R. L. Urbanke, "Approaching the AWGN channel capacity without active shaping," in Proc. IEEE Int. Symp. on Information Theory, June/July 1997, p. 374.

[10] L. Duan, "Approaching the capacity of the AWGN channel via multilevel coding," Ph.D. dissertation, Washington University, 1997.

[11] U. Wachsmann, R. F. H. Fischer, and J. B. Huber, "Multilevel codes: theoretical concepts and practical design rules," IEEE Trans. Inform. Theory, vol. 45, pp. 1361-1391, July 1999.

[12] H. S. Cronie, "Sparse graph codes for multilevel modulation with signal shaping," in Proc. IEEE Int. Symp. on Information Theory, Sept. 2005, pp. 1617-1621.

[13] T. J. Richardson, M. A. Shokrollahi, and R. L. Urbanke, "Design of capacity-approaching irregular low-density parity-check codes," IEEE Trans. Inform. Theory, vol. 47, pp. 619-637, Feb. 2001. 